\title{
The underlying structures of sentences are not the primary units of speech processing: A reinterpretation of Bever, Lackner and Kirk's findings
}

\author{
THOMAS C. TOPPINO* \\ University of New Mexico, Albuquerque, New Mexico 87106
}

\begin{abstract}
Results of an experiment by T. Bever, J. Lackner, and R. Kirk were originally interpreted to imply that listeners segment speech on the basis of underlying sentence propositions rather than on the basis of surface constituent structure. However, a reexamination of the data reveals that the results do not support such a conclusion. If anything, the data actually offer more support for the surface structure hypothesis which was originally rejected.
\end{abstract}

When a nonspeech sound (e.g., a click) is heard during a sentence, Ss tend to report the click as having occurred closer to the main surface constituent boundary than it actually occurred (Fodor \& Bever, 1965; Garrett, Bever, \& Fodor, 1966). This finding was originally interpreted to mean that a listener segments speech on the basis of surface constituent structure. However, Bever, Lackner, and Kirk (1969) observed that, in the original experiments, the surface breaks which attracted clicks were usually the boundaries of underlying sentence propositions as well. In Experiment II of their paper, Bever, Lackner, and Kirk used the same procedure with stimulus sentences in which surface boundaries were separate from underlying boundaries. They interpreted their data as showing that clicks were attracted to underlying clause boundaries rather than to surface constituent boundaries. Therefore, they concluded that deep structure sentence propositions were the "primary units of immediate speech processing [p. 225]."

The purpose of this paper is to show that Bever, Lackner, and Kirk misinterpreted their data and that their findings do not support a primary effect of deep structure propositions in sentence processing.

Bever, Lackner, and Kirk used three types of sentences, but the critical comparisons involved the direction of subjective click displacements in sentences with noun phrase complement clauses and verb phrase complement clauses. For example, the following sentences were employed:

(1) Noun phrase complement: (1 The corrupt police can't bear $\left.\left(2 \text { the criminals to confess }{ }_{2}\right)_{1}\right)$.

(2) Verb phrase complement: (1 The corrupt police can't force the criminals $\left.\left(2 \text { to confess }{ }_{2}\right)_{1}\right)$.

* Requests for reprints should be sent to Thomas C. Toppino. Department of Psychology, University of New Mexico, Abuquerque, New Mexico 87131.
The authors argue that these sentences have identical surface structures but different underlying structure propositions (marked by parentheses). Hence, differences between the two sentences in click location errors can be attributed to the influence of underlying structure alone. Clicks were objectively placed either in the main verb (e.g., bear or force) or in the following noun (or noun phrase). The critical prediction involved the proportion of errors in which the click was displaced toward the point between the verb and the subsequent noun phrase. Specifically, the underlying structure hypothesis predicts that clicks will be displaced between the verb and the noun phrase more often for noun phrase complement sentences than for verb phrase complement sentences. In noun phrase sentences, the majority of clicks objectively placed in the verb should be postposed (displaced to the right) and the majority of clicks objectively placed in the noun should be preposed (displaced to the left) because the underlying break occurs between the verb and the noun. On the other hand, in verb phrase sentences, the majority of clicks in both objective positions should be postposed because the underlying break occurs after the object noun. Thus, fewer clicks should be located between the verb and noun phrase for verb phrase sentences.

The relevant findings are presented in Table 1, which is adapted from Bever, Lackner, and Kirk (1969). The overall results seem to support the underlying structure hypothesis because the proportion of clicks attracted to the point between the verb and the subsequent noun is smaller in verb phrase sentences than in noun phrase sentences. On the basis of these overall results, Bever et al concluded in favor of the underlying structure hypothesis. However, a close look at Table 1 will reveal that the overall results were obtained for exactly the wrong reasons. In verb phrase sentences, the majority of clicks in both objective positions are preposed, although it was predicted that they should be postposed. This pattern of results is interesting because it indicates that, 
Table 1

Proportion of Location Errors of Equal Magnitude into Position Between Verb and Noun Phrase in Experimental Sentences: Experiment II

\begin{tabular}{ccccc}
\hline $\begin{array}{c}\text { Complement } \\
\text { Sentences }\end{array}$ & \multicolumn{2}{c}{ Click Objectively } & Over- \\
in Verb & in Noun & all \\
\hline 1 Noun Phrase & .8 & .8 & .8 \\
2 & Verb Phrase & .4 & .7 & .6 \\
\hline
\end{tabular}

in verb phrase sentences, clicks in both objective locations were displaced toward the major surface structure break which occurs between the subject noun phrase and the main verb.

For noun phrase sentences, the obtained pattern of click displacements confirms Bever et al's predictions. However, Chapin, Smith, and Abrahamson (1972) have argued convincingly that the surface structures of noun phrase sentences and verb phrase sentences are not equivalent. A major break occurs between the main verb and the subsequent noun in noun phrase sentences, but not in verb phrase sentences. Thus, the click displacement effects in noun phrase sentences can also be interpreted as a function of surface rather than underlying boundaries.

In summary, it appears that Bever, Lackner, and Kirk's data do not necessarily support their conclusions. If anything, their data may offer more support for the effect of surface structure constituents than for the effect of underlying sentence propositions.

\section{REFERENCES}

Bever, T., Lackner, J., \& Kirk, R. The underlying structures of sentences are the primary units of immediate speech processing. Perception \& Psychophy sics, 1969, 5, 225-234.

Chapin, P., Smith, T., \& Abrahamson, A. Two factors in perceptual segmentation of speech. Journal of Verbal Learning \& Verbal Behavior, 1972, 11, 164-173.

Fodor, J., \& Bever, T. The psychological reality of linguistic segments. Journal of Verbal Learning \& Verbal Behavior, $1965,4,414-420$.

Garrett, M., Bever, T., \& Fodor, J. The active use of grammaer in speech perception. Perception \& Psychophysics, 1966, 1, 30-32.

(Received for publication April 30, 1973; accepted January 14, 1974.) 